\title{
SOME RIGIDITY RESULTS FOR SPATIALLY CLOSED SPACETIMES
}

\author{
GREGORY J. GALLOWAY \\ Department of Mathematics and Computer Science \\ University of Miami, Coral Gables, FL 33124, U.S.A. \\ E-mail: galloway@math.miami.edu
}

1. Introduction. The geometric maximum principle for (smooth) hypersurfaces is a basic tool in Riemannian geometry. A corresponding maximum principle for spacelike hypersurfaces has become a useful tool in Lorentzian geometry, as well. These geometric maximum principles are consequences of the analytic maximum principle for second order linear elliptic PDE's (see e.g., [GT]). More closely related to the considerations of this paper is Calabi's $[\mathrm{C}]$ extension of the Hopf maximum principle to $C^{0}$ functions which are sub or super solutions in the sense of support functions. In recent joint work with Lars Andersson and Ralph Howard [AGH], we established a strong maximum principle for weak (in the sense of support functions) sub and super solutions of second order quasi-linear elliptic PDE's (such as the mean curvature equation). This generalizes Calabi's result and yields, as a geometric consequence, a maximum principle for rough (nonsmooth) spacelike hypersurfaces in Lorentzian manifolds. Such hypersurfaces arise naturally in applications as level sets of certain distance functions or Busemann functions, etc.

The maximum principle for rough spacelike hypersurfaces obtained in $[\mathrm{AGH}]$ has been applied to a number of situations ([AGH], $[\mathrm{AH}])$. In particular, it provides an especially natural and conceptually transparent proof of the Lorentzian splitting theorem ([E2], $[\mathrm{G} 2],[\mathrm{N}])$. In this paper we discuss a specialized version of the geometric maximum principle obtained in $[\mathrm{AGH}]$, in which one hypersurface is smooth and the other is rough. Some applications of the standard geometric maximum principle and this rough version are considered. In particular, we obtain a rigidity result for rough spacelike hypersurfaces that generalizes a result of Gerhardt ([GC], see also Eschenburg [E3]) concerning the uniqueness of compact maximal (i.e., mean curvature zero) spacelike hypersurfaces in globally hyperbolic spacetimes. We establish a connection between this rigidity result and a well-known splitting conjecture for spatially closed spacetimes (cf., Bartnik [B2,

1991 Mathematics Subject Classification: Primary 53C50 and 83C75.

The paper is in final form and no version of it will be published elsewhere. 
Conjecture 2]). We give a proof of this conjecture subject to an additional assumption which is weaker than other such assumptions considered previously in the literature (cf., [G1], [B2], [EG]). In fact, we present two proofs, one based on the aforementioned rigidity result, and the other based on the Lorentzian splitting theorem. The latter proof involves a generalization of the main result in [EG].

In Section 2 we discuss the maximum principle for smooth spacelike hypersurfaces and consider some consequences. In Section 3 we present the rough - smooth version of the maximum principle and prove the rigidity result for rough spacelike hypersurfaces mentioned above. The connection with the splitting conjecture is presented in Section 4 .

2. Hypersurface rigidity - the smooth case. We begin by fixing some notation and terminology. By a spacetime we mean a connected time orientable Lorentzian manifold $(M, g)$, with $\operatorname{dim} M \geq 2$. For causal theoretic notions such as $I^{ \pm}, J^{ \pm}, D^{ \pm}, H^{ \pm}$, we refer the reader to Hawking and Ellis [HE]. (Unless otherwise stated, we adhere to the conventions of $[\mathrm{HE}]$.$) Although it is not required for some of the results we consider, for$ convenience we will restrict attention to globally hyperbolic spacetimes.

Let $\Sigma$ be a smooth spacelike hypersurface in a spacetime $M$, i.e., a smooth codimension one submanifold of $M$ with everywhere timelike normal. Let $H$ denote the mean curvature of $\Sigma$. Hence, $H=\operatorname{tr} b_{N}=g^{i j} b_{i j}$, where $b_{N}$ is the second fundamental form of $\Sigma$ with respect to the future pointing unit normal vector field $N$ along $\Sigma$. We choose our sign convention so that $H=+\operatorname{div}_{\Sigma} N$. With respect to these conventions, the hyperboloid $x^{0}=+\sqrt{1+\sum\left(x^{\alpha}\right)^{2}}$ in Minkowski space has positive mean curvature.

Below, in the statement of the geometric maximum principle for spacelike hypersurfaces, we adopt the following terminology. Let $\Sigma_{1}$ and $\Sigma_{2}$ be smooth spacelike hypersurfaces in a spacetime $M$ that meet at a point $p \in M$. We say that $\Sigma_{2}$ is locally to the future of $\Sigma_{1}$ near $p$ provided for some neighborhood $\mathrm{U}$ of $p$ in which $\Sigma_{1}$ is a partial Cauchy surface (i.e., acausal and edgeless), $\Sigma_{2} \cap U \subset J^{+}\left(\Sigma_{1}, U\right)$.

Theorem 2.1 (The Maximum Principle for Spacelike Hypersurfaces). Let $\Sigma_{1}$ and $\Sigma_{2}$ be smooth spacelike hypersurfaces in a spacetime $M$ which meet at a point $p \in M$, such that $\Sigma_{2}$ is locally to the future of $\Sigma_{1}$ near $p$. Suppose that the mean curvature of $\Sigma_{1}$ and the mean curvature of $\Sigma_{2}$ satisfy, $H_{\Sigma_{2}} \leq a \leq H_{\Sigma_{1}}$, for some constant a. Then $\Sigma_{1}$ and $\Sigma_{2}$ agree in a neighborhood of $p$.

By introducing special coordinates and using the fact that the mean curvature operator is elliptic, the proof can be reduced to an application of the Hopf maximum principle (or a slight variant, thereof; see e.g., [GT, p. 35]). A proof by this approach in the case of minimal hypersurfaces in Euclidean space may be found in [S]. An alternative proof of Theorem 2.1, which uses more directly the geometry of spacetime, is given in [E3].

We consider an application of Theorem 2.1. Cosmological models based on classical general relativity typically begin with a big bang, and either expand indefinitely, or reach a point of maximum expansion and then evolve to a big crunch. Provided appropriate energy conditions are satisfied, one cannot have models which initially contract and then later expand. Such behavior is ruled out by the following well-known result of Brill and Flaherty $[\mathrm{BF}]$. 
THEOREM 2.2. Let $M$ be a globally hyperbolic spacetime which satisfies the energy condition, $\operatorname{Ric}(X, X)=R_{i j} X^{i} X^{j}>0$ for all timelike vectors $X$. Suppose $\Sigma_{1}$ and $\Sigma_{2}$ are compact smooth spacelike hypersurfaces in $M$ such that $H_{\Sigma_{1}} \leq 0 \leq H_{\Sigma_{2}}$. Then $\Sigma_{2}$ cannot enter the timelike future of $\Sigma_{1}, \Sigma_{2} \cap I^{+}\left(\Sigma_{1}\right)=\emptyset$, i.e., $\Sigma_{2} \subset J^{+}\left(\Sigma_{1}\right)$.

Remark. $\Sigma_{1}$ and $\Sigma_{2}$ are necessarily Cauchy hypersurfaces (cf. $\left.[\mathrm{B}+]\right)$.

This theorem says, in particular, that $M$ cannot contain two distinct compact maximal, i.e., mean curvature zero, spacelike hypersurfaces. The proof uses a variation of arc length argument: Assuming $\Sigma_{2} \cap I^{+}\left(\Sigma_{1}\right) \neq \emptyset$, one constructs a longest timelike geodesic $\gamma$ from $\Sigma_{1}$ to $\Sigma_{2}$. By "averaging" the second variations of arc length with respect to certain variations of $\gamma$ with end points on $\Sigma_{1}$ and $\Sigma_{2}$, a contradiction to the maximality of $\gamma$ is obtained. A related result and argument in the Riemannian setting had previously been considered by Frankel $[\mathrm{F}]$.

If the strict curvature inequality in Theorem 2.2 is replaced by the weak inequality: $\operatorname{Ric}(X, X) \geq 0$ for all timelike vectors $X$, then, of course, the conclusion need not hold. Consider, for example, the Einstein static spacetime $\left(R \times S^{3},-d t^{2} \oplus h\right)$, where $\left(S^{3}, h\right)$ is the standard round sphere. This spacetime is foliated by totally geodesic spacelike three spheres. However, Theorem 2.2 admits a rigid generalization, which we refer to here as the hypersurface rigidity theorem.

Theorem 2.3 (The Hypersurface Rigidity Theorem). Let $M$ be a globally hyperbolic spacetime which satisfies the strong energy condition, $\operatorname{Ric}(X, X) \geq 0$ for all timelike vectors $X$. Suppose $\Sigma_{1}$ and $\Sigma_{2}$ are compact connected smooth spacelike hypersurfaces in $M$ such that $H_{\Sigma_{1}} \leq 0 \leq H_{\Sigma_{2}}$. If $\Sigma_{2}$ enters the timelike future of $\Sigma_{1}, \Sigma_{2} \cap I^{+}\left(\Sigma_{1}\right) \neq \emptyset$, then the region "between" $\Sigma_{1}$ and $\Sigma_{2}, J^{+}\left(\Sigma_{1}\right) \cap J^{-}\left(\Sigma_{2}\right)$, is isometric to the Lorentzian product $\left([0, \ell] \times \Sigma_{1},-d t^{2} \oplus h\right)$, where $h$ is the induced metric on $\Sigma_{1}$ and $\ell=d\left(\Sigma_{1}, \Sigma_{2}\right)$.

Gerhardt [GC, Theorem 7.4] gave a proof of Theorem 2.3 in the case that $\Sigma_{1}$ and $\Sigma_{2}$ are maximal, with the additional assumption that there is a "barrier hypersurface". In [E3, Theorem 3] Eschenburg used the maximum principle for spacelike hypersurfaces to obtain a local version of Theorem 2.3: He considers a longest timelike geodesic segment $\gamma$ from $\Sigma_{1}$ to $\Sigma_{2}$ and obtains a splitting of a tubular neighborhood of $\gamma$. The idea of the proof is roughly as follows. Assume $\gamma$ extends from $p \in \Sigma_{1}$ to $q \in \Sigma_{2}$. By the maximality of $\gamma$, there are no focal points to $\Sigma_{1}$ along $\gamma$, (except possibly for the point $q$, a technicality that we shall overlook). One can then push a small piece $W_{1}$ of $\Sigma_{1}$ about $p$ along the normal geodesics to $\Sigma_{1}$ a distance $d\left(\Sigma_{1}, \Sigma_{2}\right)$ to obtain a spacelike hypersurface $W_{2}$ which passes through $q$ and is locally to the future of $\Sigma_{2}$ near $q$. The curvature assumption and the Raychaudhuri equation associated with the normal geodesic congruence (which governs the mean curvature of the hypersurfaces orthogonal to this congruence) imply that the mean curvature of $W_{2}$ is nonpositive. The maximum principle for spacelike hypersurfaces then implies that $W_{2}$ is actually contained in $\Sigma_{2}$, and has zero mean curvature. It now follows easily from the curvature assumption and the Raychaudhuri equation that all of the orthogonal hypersurfaces are totally geodesic. This gives a splitting of a tubular neigborhood of $\gamma$. A straightforward continuation argument yields the global splitting claimed in Theorem 2.3. 
Remarks. The assumption in Theorem 2.3 that $\Sigma_{1}$ and $\Sigma_{2}$ are compact can be weakened. It is sufficient to require that $\Sigma_{1}$ and $\Sigma_{2}$ be acausal spacelike hypersurfaces such that $J^{+}\left(\Sigma_{1}\right) \cap J^{-}\left(\Sigma_{2}\right)$ is compact. Results for Riemannian manifolds related to Theorem 2.3 have been obtained by Ichida [I] and Kasue $[\mathrm{K}]$.

In the next section we obtain a version of Theorem 2.3 that holds for rough (nonsmooth) spacelike hypersurfaces.

3. Hypersurface rigidity - the rough case. We begin by weakening the definition of a spacelike hypersurface to allow for $C^{0}$ hypersurfaces. We adopt the definition used in $[\mathrm{EG}],[\mathrm{GH}]$ and $[\mathrm{AGH}]$.

Definition 3.1. A subset $S \subset M$ is called a $C^{0}$ spacelike hypersurface provided for each point $p \in S$ there is a neighborhood $U$ of $p$ in $M$ such that $S \cap U$ is acausal and edgeless in $U$.

A $C^{0}$ spacelike hypersurface is necessarily an imbedded topological submanifold of codimension one. A smooth spacelike hypersurface, as defined in the previous section, is a $C^{0}$ spacelike hypersurface. A $C^{0}$ spacelike hypersurface which is globally acausal and edgeless is called a partial Cauchy surface.

$C^{0}$ spacelike hypersurfaces arise from natural constructions, as is illustrated in the following example.

EXAmple 3.2. Let $S$ be an acausal $C^{0}$ spacelike hypersurface in a spacetime $M$. Let $\rho:$ int $D^{+}(S) \rightarrow R$ denote Lorentzian distance to $S$,

$$
\rho(q)=\sup \{L(\gamma): \gamma \text { a future directed causal curve from } S \text { to } q\},
$$

where $L(\gamma)$ denotes the length of $\gamma$. It follows by standard arguments that $\rho$ is a continuous time function on int $D^{+}(S)$. Hence, the (nonempty) level sets of $\rho$,

$$
S_{\tau}=\left\{q \in \operatorname{int} D^{+}(S): \rho(q)=\tau\right\},
$$

are easily seen to be acausal $C^{0}$ spacelike hypersurfaces. Note that, even when $S$ is a smooth spacelike hypersurface, the $S_{\tau}$ 's will, in general, only be $C^{0}$, due to the occurence of focal points, etc.

We now extend the meaning of mean curvature inequalities to $C^{0}$ spacelike hypersurfaces. Consider two $C^{0}$ spacelike hypersurfaces $\Sigma_{1}$ and $\Sigma_{2}$ which meet at a point $p$. As in the smooth case, we say that $\Sigma_{2}$ is locally to the future of $\Sigma_{1}$ near $p$ provided for some neighborhood $U$ of $p$ in which $\Sigma_{1}$ is acausal and edgeless, $\Sigma_{2} \cap U \subset J^{+}\left(\Sigma_{1}, U\right)$.

Definition 3.3. Let $S$ be a $C^{0}$ spacelike hypersurface in a spacetime $M$, and let $a$ be a constant. We say that $S$ has mean curvature $H \leq a$ in the sense of support hypersurfaces provided for each $p \in S$ and for each $\epsilon>0$ there exists a smooth (at least $C^{2}$ ) spacelike hypersurface $W_{p, \epsilon}$ such that

(1) $W_{p, \epsilon}$ is a future support hypersurface for $S$ at $p$, i.e., $W_{p, \epsilon}$ passes through $p$ and is locally to the future of $S$ near $p$, and

(2) the mean curvature of $W_{p, \epsilon}$ at $p$ satisfies, $H_{W_{p, \epsilon}}(p) \leq a+\epsilon$. 
As a simple illustration of the definition, consider Minkowski 2-space, $M=R^{2}$ with metric $d s^{2}=d x^{2}-d y^{2}$. Then $S=\left\{(x, y): y=-\frac{1}{2}|x|\right\}$ is a $C^{0}$ spacelike hypersurface with mean curvature $H \leq 0$. The line $y=0$ may be used as the future support hypersurface at $(0,0)$.

In an analogous fashion, one can define what it means for a $C^{0}$ spacelike hypersurface to have mean curvature $H \geq a$.

R e mark. Eschenburg [E3] has introduced a related definition (which requires, in addition, a one-sided local bound on the second fundamental forms of the support hypersurfaces) in the Riemannian setting.

In $[\mathrm{AGH}]$ a maximum principle for $C^{0}$ spacelike hypersurfaces is obtained (cf., Theorem 3.6). When one of the hypersurfaces is smooth, this maximum principle specializes, as follows.

Theorem 3.4 (The Maximum Principle for Spacelike Hypersurfaces - the roughsmooth case). Let $\Sigma_{1}$ be a smooth spacelike hypersurface and $\Sigma_{2}$ be a $C^{0}$ spacelike hypersurface. Suppose that $\Sigma_{1}$ and $\Sigma_{2}$ meet at a point $p \in M$, such that $\Sigma_{2}$ is locally to the future of $\Sigma_{1}$ near $p$. Suppose, further, that the mean curvature of $\Sigma_{1}$ and the mean curvature of $\Sigma_{2}$ satisfy, $H_{\Sigma_{2}} \leq a \leq H_{\Sigma_{1}}$, for some constant a, where the first inequality is meant in the sense of support hypersurfaces. Then $\Sigma_{1}$ and $\Sigma_{2}$ agree in a neighborhood of $p$.

The proof of Theorem 3.4 above (or, more generally, Theorem 3.6 in [AGH]) involves a melding of the proof of the maximum principle for smooth spacelike hypersurfaces and the arguments of Calabi $[\mathrm{C}]$, together with certain a priori zero, first, and second order estimates.

R e m ark. In [G2], an ad hoc maximum principle for the level sets of the Lorentzian Busemann function was obtained and used to prove the Lorentzian splitting theorem. In [E3], Eschenburg established, by methods different from those used in [AGH], a roughsmooth maximum principle for hypersurfaces in Riemannian manifolds.

We now use Theorem 3.4 to establish a certain convexity property for the $C^{0}$ spacelike hypersurfaces $S_{\tau}=\{\rho=\tau\}$ introduced in Example 3.2. This result will, in turn, be used to obtain a rough version of the hypersurface rigidity theorem discussed in Section 2.

THEOREM 3.5. Let $M$ be a spacetime which obeys the strong energy condition, $\operatorname{Ric}(X, X) \geq 0$ for all timelike vectors $X$. Let $S$ be an acausal $C^{0}$ spacelike hypersurface with mean curvature $H_{S} \leq 0$ in the sense of support hypersurfaces. Let $\Sigma$ be a connected maximal (smooth with $H=0$ ) spacelike hypersurface such that $\bar{\Sigma}$ (the closure of $\Sigma$ ) is compact, acausal and contained in int $D^{+}(S)$. If edge $(\Sigma) \subset\{\rho \leq \tau\}$ then $\Sigma \subset\{\rho \leq \tau\}$.

In particular, if edge $(\Sigma) \subset S_{\tau}$ then $\Sigma$ lies in the causal past of $S_{\tau}$. Related results have been obtained in [E3] and [G2]. The proof of Theorem 3.5 makes use of the following lemma.

Lemma 3.6. Let $M$ be a spacetime which obeys the strong energy condition, $\operatorname{Ric}(X, X)$ $\geq 0$ for all timelike vectors $X$. Let $S$ be an acausal $C^{0}$ spacelike hypersurface with mean 
curvature $H_{S} \leq a$ in the sense of support hypersurfaces. Then $S_{\tau}=\{\rho=\tau\}$ has mean curvature $H_{S_{\tau}} \leq a$ in the sense of support hypersurfaces.

Pro of of Lemma 3.6. The idea is to transport the support hypersurfaces of $S$ to $S_{\tau}$. Fix $q \in S_{\tau}$. Let $\gamma:[0, \ell] \rightarrow M$ be a unit speed timelike geodesic that realizes the Lorentzian distance from $S$ to $q, L\left(\left.\gamma\right|_{[0, t]}\right)=t=d(S, \gamma(t))$ for all $t \in[0, \ell]$.

For any $\epsilon>0$, let $W=W_{p, \frac{\epsilon}{2}}$ be a future support hypersurface for $S$ at $p=\gamma(0)$, whose mean curvature at $p$ satisfies $H_{W}(p) \leq a+\frac{\epsilon}{2}$. Since $W$ is a future support hypersurface, $\gamma$ realizes the distance from $W$ to $q$. It follows that for each $t \in(0, \ell), \gamma(t)$ is not a focal point to $W$ along $\gamma$. It is possible, though, that $q=\gamma(\ell)$ is a focal point to $W$ along $\gamma$. This technical point may be handled as follows. Bend $W$ slightly towards the future to obtain a future support hypersurface $W^{\prime}$ for $S$ at $p$ such that

(1) the second fundamental form of $W^{\prime}$ at $p$ is strictly greater than that of $W$ (i.e., the difference of second fundamental forms is positive definite), and

(2) the mean curvature of $W^{\prime}$ at $p$ satisfies, $H_{W^{\prime}}(p) \leq a+\epsilon$.

It then follows by elementary index formula techniques (or see [E1, Lemma 3.1]) that for each $t \in(0, \ell], \gamma(t)$ is not a focal point to $W^{\prime}$ along $\gamma$. This implies that the normal exponential map along $W^{\prime}$ acts as a diffeomorphism on a neighborhood of the preimage of $\gamma$. Hence, by shrinking $W^{\prime}$, if necessary, we can push $W^{\prime}$ along the normal geodesics to $W^{\prime}$ at unit speed a proper time $\ell$ to obtain a smooth spacelike hypersurface $V_{q, \epsilon}$, which will be locally to the future of $S_{\tau}$ at $q$.

Let $H(t)$ be the mean curvature at $\gamma(t)$ of the smooth spacelike hypersurface passing through $\gamma(t)$ and orthogonal to the normal geodesics of $W^{\prime}$. The Raychaudhuri equation with respect to this congruence of normal geodesics and the curvature assumption imply that $H=H(t)$ satisfies, $H^{\prime}(t) \leq 0$ for all $t \in[0, \ell]$. It follows that the mean curvature of $V_{q, \epsilon}$ at $q$ satisfies, $H_{V_{q, \epsilon}}(q) \leq a+\epsilon$. Since $q \in S_{\tau}$ and $\epsilon>0$ were chosen arbitrarily, this shows that $S_{\tau}$ has mean curvature $H_{S_{\tau}} \leq a$ in the sense of support hypersurfaces.

Pro of of Theorem 3.5. Suppose the conclusion does not hold. Then $\left.\rho\right|_{\Sigma}$ achieves an interior maximum $\tau_{0}=\rho\left(x_{0}\right)>\tau$ at some point $x_{0} \in \Sigma$. By Lemma 3.6, $S_{\tau_{0}}$ has mean curvature $H_{S_{\tau_{0}}} \leq 0$ in the sense of support hypersurfaces. Moreover, $S_{\tau_{0}}$ is locally to the future of $\Sigma$ near $x_{0}$. Theorem 3.4 then implies that $\Sigma$ and $S_{\tau_{0}}$ agree in a neighborhood of $x_{0}$. By a straightforward continuation argument, $\Sigma \subset S_{\tau_{0}}$. But, since $\tau_{0}>\tau$, this contradicts the assumption edge $(\Sigma) \subset\{\rho \leq \tau\}$.

Theorem 3.5 is now used to prove a rough version of the hypersurface rigidity theorem.

Theorem 3.7 (The Hypersurface Rigidity Theorem - the rough case). Let $M$ be a globally hyperbolic spacetime which satisfies the strong energy condition, $\operatorname{Ric}(X, X) \geq 0$ for all timelike vectors $X$. Suppose $\Sigma_{1}$ and $\Sigma_{2}$ are acausal $C^{0}$ spacelike hypersurfaces in $M$ such that $J^{+}\left(\Sigma_{1}\right) \cap J^{-}\left(\Sigma_{2}\right)$ is compact. Suppose further that $H_{\Sigma_{1}} \leq 0 \leq H_{\Sigma_{2}}$ in the sense of support hypersurfaces. If $\Sigma_{2}$ enters the timelike future of $\Sigma_{1}, \Sigma_{2} \cap I^{+}\left(\Sigma_{1}\right) \neq \emptyset$, then $\Sigma_{1}$ and $\Sigma_{2}$ are smooth and compact, and $J^{+}\left(\Sigma_{1}\right) \cap J^{-}\left(\Sigma_{2}\right)$ is isometric to the Lorentzian product $\left([0, \ell] \times \Sigma_{1},-d t^{2} \oplus h\right)$, where $h$ is the induced metric on $\Sigma_{1}$ and $\ell=d\left(\Sigma_{1}, \Sigma_{2}\right)$. 
Proof. Since $J^{+}\left(\Sigma_{1}\right) \cap J^{-}\left(\Sigma_{2}\right)$ is compact, we can find points $p \in \Sigma_{1}$ and $q \in \Sigma_{2}$ such that $d(p, q)=d\left(\Sigma_{1}, \Sigma_{2}\right)=\sup \left\{d(x, y): x \in \Sigma_{1}, y \in \Sigma_{2}\right\}$. Let $\gamma:[0, \ell] \rightarrow M$ be a maximal unit speed timelike geodesic from $p$ to $q$.

Using the global hyperbolicity of $M$ and the compactness of $J^{+}\left(\Sigma_{1}\right) \cap J^{-}\left(\Sigma_{2}\right)$, one checks that $J^{+}\left(\Sigma_{1}\right) \cap J^{-}\left(\Sigma_{2}\right)=D^{+}\left(\Sigma_{1}\right) \cap D^{-}\left(\Sigma_{2}\right)$. Consider the distance functions $\rho_{1}$ and $\rho_{2}$ to $\Sigma_{1}$ and $\Sigma_{2}$, respectively, defined as follows:

$$
\rho_{1}: \operatorname{int} D^{+}\left(\Sigma_{1}\right) \cap D^{-}\left(\Sigma_{2}\right) \rightarrow R, \quad \rho_{1}(x)=d\left(\Sigma_{1}, x\right),
$$

and

$$
\rho_{2}: \operatorname{int} D^{+}\left(\Sigma_{1}\right) \cap D^{-}\left(\Sigma_{2}\right) \rightarrow R, \quad \rho_{2}(x)=d\left(x, \Sigma_{2}\right) .
$$

The reverse triangle inequality and the maximality of $\gamma$ imply

$$
\rho_{1}+\rho_{2} \leq \ell \quad \text { and } \quad \rho_{1}+\rho_{2}=\ell \quad \text { along } \gamma \text {. }
$$

Consider the $C^{0}$ spacelike hypersurfaces, $S_{1}=\left\{\rho_{1}=\frac{\ell}{2}\right\}$ and $S_{2}=\left\{\rho_{2}=\frac{\ell}{2}\right\}$. By (3.1), $S_{1}$ and $S_{2}$ pass through the point $p=\gamma\left(\frac{\ell}{2}\right)$, and $S_{1}$ is locally to the future of $S_{2}$ near $p$. Lemma 3.6 and its time dual imply that $H_{S_{1}} \leq 0$ and $H_{S_{2}} \geq 0$ in the sense of support hypersurfaces.

By an argument similar to that used in [G2], we show that $S_{1}$ and $S_{2}$ agree near $p$ along a maximal (smooth with $H=0$ ) spacelike hypersurface. Let $B$ be a small coordinate ball in $S_{1}$ centered at $p$. Then, by a basic existence result of Bartnik [B1, Theorem 4.1], provided $B$ is sufficiently small, $\partial B$ can be spanned by a maximal spacelike hypersurface. More precisely, there exists an acausal maximal spacelike hypersurface $\Sigma \subset \operatorname{int} D^{+}\left(\Sigma_{1}\right) \cap D^{-}\left(\Sigma_{2}\right)$ intersecting $\gamma$, such that $\bar{\Sigma}$ is compact and edge $(\Sigma)=\partial B$. By Theorem 3.5 and its time dual, $\Sigma \subset\left\{\rho_{1} \leq \frac{\ell}{2}\right\} \cap\left\{\rho_{2} \leq \frac{\ell}{2}\right\}$. In particular, this forces $\Sigma$ to meet $\gamma$ at $p$. Hence, $S_{1}$ is locally to the future of $\Sigma$ near $p$ and $S_{2}$ is locally to the past of $\Sigma$ near $p$. By Theorem 3.4 and its time dual, $S_{1}, S_{2}$ and $\Sigma$ agree in a neighborhood of $p$. Reducing the size of $\Sigma$ if necessary, we conclude that

$$
\rho_{1}=\rho_{2}=\frac{\ell}{2} \text { along } \Sigma \text {. }
$$

We now show that the normal exponential map along $\Sigma$ provides a splitting of a tubular neighborhood of $\gamma$. For each $x \in \Sigma$, there exists a future directed unit speed timelike geodesic $\gamma_{x}:\left[0, \frac{\ell}{2}\right] \rightarrow M$ which realizes the distance from $x$ to $\Sigma_{2}, L\left(\left.\gamma_{x}\right|_{\left[t, \frac{\ell}{2}\right]}\right)=$ $\frac{\ell}{2}-t=\rho_{2}\left(\gamma_{x}(t)\right)$, for all $t \in\left[0, \frac{\ell}{2}\right]$. It then follows that each initial segment of $\gamma_{x}$ maximizes to $\Sigma, L\left(\left.\gamma_{x}\right|_{[0, t]}\right)=t=d\left(\Sigma, \gamma_{x}(t)\right)$ for all $t \in\left[0, \frac{\ell}{2}\right]$ (otherwise, there would exist a timelike curve from $\Sigma$ to $\Sigma_{2}$ with length greater than $\frac{\ell}{2}$, contradicting (3.2)). This implies the following:

(1) $\gamma_{x}$ meets $\Sigma$ orthogonally.

(2) For each $t \in\left[0, \frac{\ell}{2}\right), \gamma_{x}(t)$ is not a focal point to $\Sigma$ along $\gamma_{x}$.

(3) If $x \neq y$ then $\left.\gamma_{x}\right|_{\left[0, \frac{\ell}{2}\right)}$ and $\left.\gamma_{y}\right|_{\left[0, \frac{\ell}{2}\right)}$ do not intersect.

Consider the normal exponential map $\Phi:\left[0, \frac{\ell}{2}\right) \times \Sigma \rightarrow M, \Phi(t, x)=\exp _{x}(t N)=$ $\gamma_{x}(t)$, where $N$ is the future pointing timelike unit normal vector field along $\Sigma$. The properties (1) - (3) above guarantee that $\Phi$ is a diffeomorphism onto its image. For each $t \in\left[0, \frac{\ell}{2}\right), \Sigma(t)=\Phi(\{t\} \times \Sigma)$ is a smooth spacelike hypersurface orthogonal to the 
normal geodesics of $\Sigma$. Let $B(t)$ and $H(t)$ denote the second fundamental form and mean curvature, respectively, of $\Sigma(t)$. Extend $N$ to be the future pointing unit normal vector field to the $\Sigma(t)$ 's.

The mean curvature function $H=H(t)$ of the foliation $\{\Sigma(t)\}$ obeys the following evolution equation (essentially the Raychaudhuri equation associated to the congruence of normal geodesics):

$$
\frac{\partial H}{\partial t}=-\operatorname{Ric}(N, N)-|B|^{2} .
$$

The curvature assumption and (3.3) imply that $\frac{\partial H}{\partial t} \leq 0$. Since $H(0)=0$, we conclude that $H(t) \leq 0$ for all $t \in\left[0, \frac{\ell}{2}\right)$. On the other hand, since $\Sigma(t) \subset\left\{\rho_{2}=\frac{\ell}{2}-t\right\}$, the time dual of Lemma 3.6 implies that $H(t) \geq 0$. Thus, $H(t)=0$ for all $t \in\left[0, \frac{\ell}{2}\right)$. Substitution into (3.3) then gives, $B(t)=0$ for all $t \in\left[0, \frac{\ell}{2}\right)$ (i.e., each $\Sigma(t)$ is totally geodesic). Hence, $N$ is a parallel vector field and $\Phi:\left[0, \frac{\ell}{2}\right) \times \Sigma \rightarrow M$ is an isometry onto its image.

We now observe that this isometry extends to $t=\frac{\ell}{2}$. The vanishing of the second fundamental forms of the $\Sigma(t)$ 's implies that for each $x \in \Sigma$, the point $\Phi\left(\frac{\ell}{2}, x\right)=\gamma_{x}\left(\frac{\ell}{2}\right)$ cannot be a focal point to $\Sigma$ along $\gamma_{x}$ (for if it were, one would have $\liminf _{t \rightarrow \frac{\ell}{2}} H(t)=$ $-\infty)$. Thus for each $x \in \Sigma$, there exists a neighborhood $W_{x} \subset \Sigma$ of $x$ such that $y \in$ $W_{x} \mapsto \Phi\left(\frac{\ell}{2}, y\right) \in M$ is a smooth spacelike imbedding. It follows that $\Phi\left(\left\{\frac{\ell}{2}\right\} \times \Sigma\right) \subset \Sigma_{2}$ is a smooth spacelike hypersurface containing the point $q$. Hence, each $\gamma_{x}$ meets $\Sigma_{2}$ orthogonally, from which it follows that if $x \neq y$ then $\gamma_{x}\left(\frac{\ell}{2}\right) \neq \gamma_{y}\left(\frac{\ell}{2}\right)$. These observations imply that $\Phi:\left[0, \frac{\ell}{2}\right] \times \Sigma \rightarrow M$ is an isometry onto its image. Finally, by considering the time-dual of the above arguments (e.g., by considering maximal segments from $x \in \Sigma$ to $\left.\Sigma_{1}\right)$ we conclude that the normal exponential map along $\Sigma, \Phi:\left[-\frac{\ell}{2}, \frac{\ell}{2}\right] \times \Sigma \rightarrow M$, is an isometry onto its image, such that $\Phi\left(\left\{-\frac{\ell}{2}\right\} \times \Sigma\right) \subset \Sigma_{1}$ and $\Phi\left(\left\{\frac{\ell}{2}\right\} \times \Sigma\right) \subset \Sigma_{2}$.

Thus, summarizing and slightly reformulating the above, given the timelike geodesic $\gamma:[0, \ell] \rightarrow M$ from $p \in \Sigma_{1}$ to $q \in \Sigma_{2}$, of length $\ell=d\left(\Sigma_{1}, \Sigma_{2}\right)$, there exists a neighborhood $W_{1} \subset \Sigma_{1}$ of $p$ which is a smooth spacelike hypersurface such that the normal exponential map along $W_{1}, \Psi:[0, \ell] \times W_{1} \rightarrow \Psi\left([0, \ell] \times W_{1}\right) \subset J^{+}\left(\Sigma_{1}\right) \cap J^{-}\left(\Sigma_{2}\right), \Psi(t, x)=\exp _{x} t N_{1}$, is an isometry. Moreover, $\Psi\left(\{0\} \times W_{1}\right)=W_{1}$ and $W_{2}:=\Psi\left(\{\ell\} \times W_{1}\right) \subset \Sigma_{2}$ is a smooth spacelike hypersurface containing $q$.

The process of extending this local splitting to the global splitting claimed in Theorem 3.7 is fairly straightforward. Let $A$ be the component of $\Sigma_{1}$ containing $p$. Let,

$U=\left\{x \in A: \exists\right.$ a timelike geodesic $\alpha$ from $x$ to $\Sigma_{2}$ of length $\left.\ell=d\left(\Sigma_{1}, \Sigma_{2}\right)\right\}$.

By continuity properties of geodesics, $U$ is closed in $A$, and by the local isometries established above, $U$ is open in $A$. Hence, $U=A$. It follows, by piecing these local isometries together, that $A$ is a smooth spacelike hypersurface, and the normal exponential along $A, \Psi:[0, \ell] \times A \rightarrow \Psi([0, \ell] \times A) \subset J^{+}\left(\Sigma_{1}\right) \cap J^{-}\left(\Sigma_{2}\right), \Psi(t, x)=\exp _{x} t N_{1}$, is an isometry such that $\Psi(\{0\} \times A)=A$, and $\Psi(\{\ell\} \times A)$ is a smooth spacelike hypersurface contained in $\Sigma_{2}$. The compactness of $J^{+}\left(\Sigma_{1}\right) \cap J^{-}\left(\Sigma_{2}\right)$ forces $A$ to be edgeless, and hence closed as a subset of $M$. Since $A \subset J^{+}\left(\Sigma_{1}\right) \cap J^{-}\left(\Sigma_{2}\right), A$ is compact and Cauchy (by [B+]). Hence, to avoid an acausality violation, $\Sigma_{1}$ must consist of a single component, i.e., $\Sigma_{1}=A$. Theorem 3.7 now follows easily. 
4. The splitting conjecture for spatially closed spacetimes. The classical Hawking-Penrose singularity theorems (cf., [HE]) establish the existence of singularities, expressed in terms of incomplete causal geodesics, in large generic classes of spacetimes. In [GR2] Geroch put forth the conjectural point of view that spatially closed spacetimes obeying reasonable energy conditions should fail to be singular only under exceptional circumstances. We refer especially to the discussion on p. 266 in [GR2] (in which Geroch remarks on the existence of certain geodesically complete flat spacetimes), the figure on p. 266, and Problem 6 posed on p. 288. In the early 80's, Yau formulated this problem in more differential geometric terms, by posing the problem of establishing the rigidity of the Hawking-Penrose singularity theorems. This has led to the following explicit conjecture (stated as Conjecture 2 in Bartnik [B2]).

CONJECTURE. Let $M$ be a spacetime which contains a compact Cauchy surface and obeys the strong energy condition, $\operatorname{Ric}(X, X) \geq 0$ for all timelike vectors $X$. If $M$ is timelike geodesically complete, then $M$ splits isometrically into the product $\left(R \times V,-d t^{2} \oplus\right.$ $h)$, where $(V, h)$ is a compact Riemannian manifold.

In other words, $M$ either is singular or exceptional (a Lorentzian product). For a more detailed account of the background relating to this conjecture, we refer the reader to [BEE, Chapter 14].

Remark. Recall, a $C^{0}$ spacelike hypersurface $S$ in $M$ is a Cauchy surface if and only if $D(S)=M . S$ is a compact Cauchy surface for $M$ if and only if $M$ is globally hyperbolic and $S$ is compact $([\mathrm{B}+])$.

There have been basically two approaches to proving the conjecture. One approach, first considered by Geroch [GR1], is to establish the existence of a compact maximal spacelike hypersurface; the conjecture is well-known to hold in this case (see e.g., [B2], [G3]). The other approach, originally advocated by Yau, is to establish the existence of a timelike line (i.e., a globally maximal inextendible timelike geodesic). The conjecture would then follow from the Lorentzian splitting theorem (see, e.g. [BEE, Chapter 14] for an account of this theorem). The difficulty with this latter approach is that, although there is a standard procedure for constructing a causal (timelike or null) line in a spacetime with compact Cauchy surface, the line need not be timelike [EhG]. The conjecture has been proved subject to the addition of various "no horizon" type conditions ([G1], [B2], [EG]). Below we present a proof of the conjecture subject to a milder additional condition.

Let $S$ be a $C^{0}$ spacelike hypersurface in a spacetime $M$. A future inextendible timelike geodesic $\gamma:[0, a) \rightarrow M, a \in(0, \infty]$, is called a future $S$-ray provided it maximizes distance to $S$, i.e. provided, $L\left(\left.\gamma\right|_{[0, t]}\right)=d(S, \gamma(t))$ for all $t \in[0, a)$. One defines a past $S$-ray time-dually. If $S$ is compact, it aways admits a past and future $S$-ray.

THEOREM 4.1. Let $M$ be a spacetime which contains a compact Cauchy surface $S$ and obeys the strong energy condition. If $M$ is timelike geodesically complete, and contains a future $S$-ray $\gamma$ and a past $S$-ray $\eta$ such that $I^{-}(\gamma) \cap I^{+}(\eta) \neq \emptyset$ then $M$ splits as in the conjecture. 
We actually give two proofs of Theorem 4.1. The first proof is an application of Theorem 3.7, and the second is an application of the Lorentzian splitting theorem. Both proofs make use of the lemma presented below. Given an acausal $C^{0}$ spacelike hypersurface $S$ in a spacetime $M$, let $\rho: D(S) \rightarrow R$, denote the signed distance function from $S$,

$$
\rho(x)= \begin{cases}d(S, x), & \text { for } x \in D^{+}(S) \\ -d(x, S), & \text { for } x \in D^{-}(S)\end{cases}
$$

We note that $\rho$ is a continuous time function on $D(S)$.

Lemma 4.2. Let $M$ be a timelike geodesically complete spacetime with compact Cauchy surface $S$. Then for each $c \in R, S_{c}=\{\rho=c\}$ is a compact Cauchy surface for $M$.

Proof. We prove the lemma for $c \geq 0$; the proof for $c \leq 0$ follows by time-dual arguments. Since $D(S)=M, \rho$ is a continuous time function on $M$, and hence $S_{c}, c \geq 0$, is a partial Cauchy surface contained in $J^{+}(S)$. (Note that the future completeness of $M$ guarantees that $S_{c} \neq \emptyset$.)

Let $x$ be any point in $J^{+}\left(S_{c}\right)$, and let $\eta:[0, \infty) \rightarrow M$ be any past inextendible causal curve in $M$ starting at $x$. Since $S$ is Cauchy, $\eta$ enters $I^{-}(S)$. Hence, $\rho \circ \eta(t)$ is a continuous decreasing function such that $\rho \circ \eta(0) \geq c$ and $\rho \circ \eta(t)<0$ for $t$ sufficiently large. Thus, $\rho \circ \eta\left(t_{0}\right)=c$ for some $t_{0}$, i.e., $\eta$ meets $\Sigma_{c}$.

Now, let $x$ be any point in $J^{-}\left(S_{c}\right)$, and let $\eta:[0, \infty) \rightarrow M$ be any future inextendible causal curve in $M$ starting at $x$. $\eta$ either starts in or eventually enters $J^{+}(S)$. Let $\gamma_{j}$ be a longest timelike geodesic segment from $S$ to $q_{j}=\eta\left(t_{j}\right) \in J^{+}(S), t_{j} \rightarrow \infty$. By a standard limit curve argument, a subsequence $\left\{\gamma_{j_{k}}\right\}$ converges to a future inextendible, and hence, by assumption, future complete timelike geodesic $S$-ray $\gamma$. Parameterizing $\gamma:[0, \infty) \rightarrow M$ with respect arc length, we have $\rho(\gamma(t))=t$ for all $t \in[0, \infty)$, which implies that $\gamma$ meets $S_{c}$. It follows that for $j_{k}$ sufficiently large, $\gamma_{j_{k}}$ meets $S_{c}$, which can happen only if $\eta$ meets $S_{c}$. We conclude from these arguments that $S_{c}$ is Cauchy surface. Since all Cauchy surfaces of $M$ are homeomorphic, $S_{c}$ is compact.

The first proof of Theorem 4.1 we give makes use of certain properties of Lorentzian Busemann functions established in [GH]. Let $\gamma:[0, \infty) \rightarrow M$ be a future complete unit speed timelike geodesic ray starting at $p=\gamma(0), L\left(\left.\gamma\right|_{[0, t]}\right)=t=d(p, \gamma(t))$, for all $t \in[0, \infty)$. The Lorentzian Busemann function $b: M \rightarrow[0, \infty]$ associated to $\gamma$ is defined as follows

$$
b(x)=\lim _{r \rightarrow \infty} r-d(x, \gamma(r)),
$$

where $d$ is the Lorentzian distance function. (For a nice introduction to Lorentzian Busemann functions, see [BEE, Chapter 14].) The regularity of the Lorentzian Busemann function is more complicated than that of its Riemannian counterpart. For instance, $b$ need not be continuous, in general. However, it will always be continuous near its associated ray. Along the ray we have $b(\gamma(t))=t$.

We now summarize some facts concerning the regularity of Lorentzian Busemann functions established in [GH] (see especially, Lemma 5.4, Corollary 5.6, Lemma 2.5, and Inequality (4-6)). 
THEOREM 4.3. Let $M$ be a future timelike geodesically complete spacetime with compact Cauchy surface $S$. Let $\gamma:[0, \infty) \rightarrow M$ be a future $S$-ray, and let $b=b_{\gamma}$ be the associated Busemann function. Then the following statements hold.

(1) $b$ is finite valued and continuous on $I^{-}(\gamma)$.

(2) The level sets $\Sigma_{c}=\{b=c\}, c \geq 0$, are partial Cauchy surfaces (i.e., acausal edgeless $C^{0}$ spacelike hypersurfaces) in $M$.

(3) If $M$ obeys the strong energy condition then $\Sigma_{c}$ has mean curvature $H_{\Sigma_{c}} \geq 0$ in the sense of support hypersurfaces.

First proof of Theorem 4.1. This proof is an application of Theorem 3.7. Fix $p=\eta\left(c_{1}\right)$ and $q=\gamma\left(c_{2}\right)$ such that $p \in I^{-}(q)$. Consider the level sets $\Sigma_{1}=\left\{b_{\eta}=c_{1}\right\}$ and $\Sigma_{2}=\left\{b_{\gamma}=c_{2}\right\}$. By Theorem 4.3 and its time-dual, $\Sigma_{1}$ and $\Sigma_{2}$ are partial Cauchy surfaces such that $H_{\Sigma_{1}} \leq 0 \leq H_{\Sigma_{2}}$ in the sense of support hypersurfaces. To apply Theorem 3.7 we need to show that $J^{+}\left(\Sigma_{1}\right) \cap J^{-}\left(\Sigma_{2}\right)$ is compact.

Let $S_{1}=\left\{\rho=-c_{1}\right\}$ and $S_{2}=\left\{\rho=c_{2}\right\}$. Lemma 4.2 implies that $S_{1}$ and $S_{2}$ are compact Cauchy surfaces. By an elementary triangle inequality argument (see e.g., [BEE]), $b_{\gamma}(x) \geq \rho(x)$ for all $x \in J^{+}(S)$. This implies that $\left.\rho\right|_{\Sigma_{2}} \leq c_{2}$, and hence that $\Sigma_{2} \subset J^{-}\left(S_{2}\right)$. Similarly, $\Sigma_{1} \subset J^{+}\left(S_{1}\right)$. It follows that $J^{+}\left(\Sigma_{1}\right) \cap J^{-}\left(\Sigma_{2}\right)$ is a subset of the compact set $K=J^{+}\left(S_{1}\right) \cap J^{-}\left(S_{2}\right)$. The equality $J^{+}\left(\Sigma_{1}\right) \cap J^{-}\left(\Sigma_{2}\right)=J^{+}\left(\Sigma_{1} \cap K\right) \cap J^{-}\left(\Sigma_{2} \cap K\right)$ is easily verified and shows that $J^{+}\left(\Sigma_{1}\right) \cap J^{-}\left(\Sigma_{2}\right)$ is compact.

Theorem 3.7 now implies that $J^{+}\left(\Sigma_{1}\right) \cap J^{-}\left(\Sigma_{2}\right)$ is isometric to a Lorentzian product $\left(\left[-c_{1}, c_{2}\right] \times \Sigma,-d t^{2} \oplus h\right)$. By letting $c_{1}, c_{2} \rightarrow \infty$, we obtain the splitting of $M$ asserted in Theorem 4.1.

Second proof of Theorem 4.1. This is an application of the Lorentzian splitting theorem. It is sufficient to establish the existence of a timelike line under appropriate circumstances. Theorem 4.1 is an immediate consequence of the Lorentzian splitting theorem and the following theorem.

THEOREM 4.4. Let $M$ be a timelike geodesically complete spacetime with compact Cauchy surface $S$. Suppose there exists a future $S$-ray $\gamma$ and a past $S$-ray $\eta$ such that $I^{-}(\gamma) \cap I^{+}(\eta) \neq \emptyset$. Then $M$ contains a timelike line.

P r o of. We fix a complete Riemannian metric $h$ on $M$. With the exception of certain limit curves which inherit a limit parameter, it will be convenient to parameterize all causal curves by arc length with respect to $h$.

We construct a causal (timelike or null) line in the most obvious way and show that it must be timelike. Choose $q_{n}=\gamma\left(t_{n}\right), t_{n} \rightarrow \infty$ and $p_{n}=\eta\left(s_{n}\right), s_{n} \rightarrow \infty$ such that $p_{n} \in I^{-}\left(q_{n}\right)$ for all $n$. Let $\sigma_{n}:\left[-a_{n}, b_{n}\right] \rightarrow M$ be a maximal timelike geodesic segment from $p_{n}$ to $q_{n}$, parameterized with respect to $h$-arc length so that $\sigma_{n}(0) \in S$. Since $p_{n}$ and $q_{n}$ diverge to infinity, and $S$ is compact, we have that $a_{n}, b_{n} \rightarrow \infty$. By using an appropriate version of the limit curve lemma (see e.g., [GH]), and passing to a subsequence if necessary, we may assume that $\left\{\sigma_{n}\right\}$ converges uniformly on compact subsets to an inextendible causal curve $\sigma:(-\infty, \infty) \rightarrow M$. By the usual arguments, the maximality of the $\sigma_{n}$ 's guarantees that $\sigma$ is a timelike or null line. We show that $\sigma$ is timelike. 
Let $\alpha_{n}=\left.\sigma_{n}\right|_{\left[0, b_{n}\right]}, \beta_{n}=-\left.\sigma_{n}\right|_{\left[0, a_{n}\right]}, \alpha=\left.\sigma\right|_{[0, \infty)}$, and $\beta=-\left.\sigma\right|_{[0, \infty)}$. We have that $\alpha_{n} \rightarrow \alpha$ uniformly on compact subsets. We now establish the following length estimate.

Claim. For each $t \in\left[0, b_{n}\right]$,

$$
L\left(\left.\alpha_{n}\right|_{[0, t]}\right) \geq \rho\left(\alpha_{n}(t)\right)-\delta,
$$

where $L$ is Lorentzian arc length and $\delta=\rho\left(p_{1}\right)+\rho\left(q_{1}\right)$.

Pro of of the claim. Using the maximality of $\sigma_{n}$, and the fact that $\gamma$ and $\eta$ are $S$-rays, we obtain

$$
\begin{aligned}
L\left(\alpha_{n}\right) & =L\left(\sigma_{n}\right)-L\left(\beta_{n}\right) \\
& \geq L\left(\left.\gamma\right|_{\left[t_{1}, t_{n}\right]}\right)+L\left(\left.\eta\right|_{\left[s_{1}, s_{n}\right]}\right)-L\left(\beta_{n}\right) \\
& =\rho\left(q_{n}\right)+\rho\left(p_{n}\right)-\delta-L\left(\beta_{n}\right) \\
& \geq \rho\left(q_{n}\right)-\delta
\end{aligned}
$$

which proves (4.1) for $t=b_{n}$.

Now, for $t \in\left[0, b_{n}\right)$, we have by (4.2),

$$
\begin{aligned}
L\left(\left.\alpha_{n}\right|_{[0, t]}\right) & =L\left(\alpha_{n}\right)-L\left(\left.\alpha_{n}\right|_{\left[t, b_{n}\right]}\right) \\
& \geq \rho\left(q_{n}\right)-d\left(\alpha_{n}(t), q_{n}\right)-\delta \\
& \geq\left(\rho\left(\alpha_{n}(t)\right)+d\left(\alpha_{n}(t), q_{n}\right)\right)-d\left(\alpha_{n}(t), q_{n}\right)-\delta \\
& =\rho\left(\alpha_{n}(t)\right)-\delta,
\end{aligned}
$$

which establishes the claim.

Letting $n \rightarrow \infty$ in (4.1) we obtain

$$
L\left(\left.\alpha\right|_{[0, t]}\right) \geq \rho(\alpha(t))-\delta \text { for all } t \in[0, \infty) .
$$

Lemma 4.2 implies that $\alpha$ must meet $S_{c}=\{\rho=c\}$ for all $c \geq 0$. It follows that $\rho(\alpha(t)) \rightarrow$ $\infty$ as $t \rightarrow \infty$. Thus, for $t$ sufficiently large, the right hand side of (4.3) is positive. This implies that $\alpha$, and, hence, $\sigma$ are timelike.

Remarks. Theorem 4.4 generalizes, in the globally hyperbolic setting, the main result in [EG]. Moreover, as in [EG], the assumption of global hyperbolicity in Theorem 4.4 can be substantially weakened. It is sufficient to assume that $S$ is a compact acausal $C^{0}$ spacelike hypersurface. Thus, Theorem 4.1 remains valid under the weaker assumption that $S$ is compact and acausal.

The following corollary to Theorem 4.1 addresses the problem of Geroch alluded to above [GR2, Problem 6, p.288].

Corollary 4.5. Let $M$ be a 4-dimensional spacetime with compact Cauchy surface $S$, which obeys the following energy condition: For each timelike vector $X \in T_{p} M$, $\operatorname{Ric}(X, X) \geq 0$ and $=0$ if and only if Ric $=0$ at $p$. If $M$ is timelike geodesically complete, and contains a future $S$-ray $\gamma$ and a past $S$-ray $\eta$ such that $I^{-}(\gamma) \cap I^{+}(\eta) \neq \emptyset$ then $M$ is flat. 
Remark. By the Einstein equation (with zero cosmological constant), the energy condition will be satisfied for Type I energy-momentum tensors [HE, p. 89], provided the energy density $\mu$ and the principal pressures $p_{\alpha}, \alpha=1,2,3$, are nonnegative.

Proof. By Theorem 4.1, $M$ splits as in the conjecture, $\left(M^{4}, g\right)=\left(R \times V^{3},-d t^{2} \oplus h\right)$. The product structure implies that $\operatorname{Ric}\left(\frac{\partial}{\partial t}, \frac{\partial}{\partial t}\right) \equiv 0$, and hence, by the energy condition, $M$ is Ricci flat. The product structure then also implies that $V$ is Ricci flat. Since $\operatorname{dim} V=$ $3, V$ is flat.

Although the Bartnik conjecture remains open, it has stimulated the development of some interesting mathematics which may ultimately play a role in its final resolution.

Acknowledgement. This paper is based in part on lectures given during the Minisemester on Mathematical Aspects in Theories of Gravitation at the Banach Center, University of Warsaw. I would like to thank the center and the organizers of the minisemseter for the hospitality and support.

\section{References}

[AGH] L. Andersson, G.J. Galloway, and R. Howard, A strong maximum principle for weak solutions of quasi-linear elliptic equations with applications to Lorentzian geometry, preprint.

$[\mathrm{AH}]$ L. Andersson and R. Howard, Rigidity results for Robertson-Walker and related spacetimes, preprint.

[B1] R. Bartnik, Regularity of variational maximal surfaces, Acta Mathematica 161 (1988), 145-181.

[B2] R. Bartnik, Remarks on cosmological spacetimes and constant mean curvature surfaces, Commun. Math. Phys. 117 (1988), 615-624.

[BEE] J.K. Beem, P.E. Ehrlich, K.L. Easley, Global Lorentzian Geometry, 2nd ed., Marcel Dekker, New York, 1996.

[BF] D. Brill and F. Flaherty, Isolated maximal hypersurfaces in spacetime, Commun. Math. Phys. 50 (1976), 157-165.

[B+] R. Budic, J. Isenberg, L. Lindblom and P.B. Yasskin, On the determination of Cauchy surfaces from intrinsic properties, Comm. Math. Phys. 61 (1978), 87-95.

[C] E. Calabi, An extension of E. Hopf's maximum principle with applications to Riemannian geometry, Duke Math. J. 25 (1957), 45-56.

[EhG] P. Ehrlich and G.J. Galloway, Timelike lines, Classical and Quantum Grav. J. (1990), 297-307.

[E1] J.-H. Eschenburg, Local convexity and nonnegative curvature - Gromov's proof of the sphere theorem, Invent. Math. 84 (1986), 507-522.

[E2] J.-H. Eschenburg, The splitting theorem for spacetimes with strong energy condition, J. Diff. Geom. 27 (1988), 477-491.

[E3] J.-H. Eschenburg, Maximum principles for hypersurfaces, Manuscripta Math. 64 (1989), 55-75.

[EG] J.-H. Eschenburg and G.J. Galloway, Lines in spacetimes, Comm. Math. Phys. 148 (1992), 209-216. 
[F] T. Frankel, On the fundamental group of a comapct minimal submanifold, Ann. Math. 83 (1966), 68-73.

[G1] G.J. Galloway, Splitting theorems for spatially closed space-times, Commun. Math. Phys. 96 (1984), 423-429.

[G2] G.J. Galloway, The Lorentzian splitting theorem without completeness assumption, J. Diff.Geom. 29 (1989), 373-387.

[G3] G.J. Galloway, Some connections between global hyperbolicity and geodesic completeness, J. Geom. Phys. 6 (1989), 127-141.

[GH] G.J. Galloway and A. Horta, Regularity of Lorentzian Busemann functions, Trans. Amer. Math. Soc. 348 (1996), 2063-2084.

[GC] C. Gerhardt, H-surfaces in Lorentzian manifolds, Commun. Math. Phys. 89 (1983), 523-553.

[GR1] R. Geroch, Singularities in closed universes, Phys. Rev. Lett. 17 (1966), 445-447.

[GR2] R. Geroch, Singularities, in: Relativity, M. Carmeli, S. Fickler and L. Witten (eds.), Plenum Press, New York, 1970, 259-291.

[GT] D. Gilbarg and N.S. Trudinger, Elliptic partial differential equations, 2nd ed., Springer-Verlag, New York, 1983.

[HE] S.W. Hawking and G.F.R. Ellis, The large scale structure of space-time, Cambridge University Press, Cambridge, 1973.

[I] R. Ichida, Riemannian manifolds with compact boundary, Yokohama Math. J. 29 (1981), 169-177.

[K] A. Kasue, Ricci curvature, geodesics and some geometric properties of Riemannian manifolds with boundary, J. Math. Soc. Japan, 35 (1983), 117-131.

[N] R.P.A.C. Newman, A proof of the splitting conjecture of S.-T. Yau, J. Diff. Geom. 31 (1990), 163-184.

[S] R. Schoen, Uniqueness, symmetry and embeddedness of minimal surfaces, J. Diff. Geom. 18 (1983), 791-804. 